\title{
False aneurysm of the common femoral vein in a footballer
}

\author{
M Karahan, S Isbir, F Baltacýoglu, M M Caglar
}

Br J Sports Med 2005;39:e8 (http://www.bjsportmed.com/cgi/content/full/39/2/e8). doi: 10.1136/bjsm.2004.012682

Traumatic false aneurysm of the femoral vein has never been reported in the English literature. The case is here reported of a footballer with a traumatic false aneurysm of the common femoral vein which was initially misdiagnosed as an arterial pseudoaneurysm. This is a very rare clinical condition, but this diagnosis should be among those considered for posttraumatic unexplained thigh pain after trauma.

$\mathrm{T}$ raumatic false aneurysm of the femoral vein has never been reported in the English literature. Patients with venous aneurysm may be completely asymptomatic or have various symptoms. ${ }^{1-3}$ A survey of the literature has suggested that traumatic venous false aneurysm is a diagnostic challenge as in the current case..$^{2-6}$ The case is presented of a footballer with a traumatic false aneurysm of the common femoral vein which was initially misdiagnosed as arterial pseudoaneurysm.

\section{CASE REPORT}

A 28 year old footballer was admitted to our hospital with pain and major swelling in his left thigh two weeks after being struck forcefully with the sole of another player's boot. He suffered swelling and excruciating pain during training. Conservative treatment had been unsuccessful. Physical examination at rest was normal except tenderness and a slight swelling of the left middle thigh. His laboratory tests were normal. Colour Doppler ultrasound imaging of the lower limb, performed after five minutes of running, revealed a pseudoaneurysm in the middle of the left superficial femoral artery. The pseudoaneurysm showed a to and fro pattern of flow during spectral examination confirming that it was not a haematoma. Digital subtraction angiography (DSA) examination showed a pseudoaneurysm of the left superficial femoral artery measuring $12 \times 9 \mathrm{~mm}$ and oriented anterolaterally (fig 1). As both the colour Doppler ultrasonography and early arterial phase DSA examinations led us to think that this aneurysm was strictly an arterial pseudoaneurysm, we did not feel the need to take late venous phase images during DSA examination. Magnetic resonance imaging with a fat saturated $\mathrm{T}^{\prime}$ weighted sequence (TR/TE, 2500/ 105 milliseconds) revealed a haematoma of $5 \times 6 \mathrm{~cm}$ with a cystic central component of $2 \times 2.5 \mathrm{~cm}$, probably a result of haematoma resorption. There was also oedema in the surrounding soft tissues extending to the subcutaneous tissue.

The patient was operated on with the diagnosis of arterial pseudoaneurysm. Doppler ultrasound examination performed under anaesthesia was normal. The superficial femoral artery from the common femoral artery to the origin of Hunter's canal was normal. There was no leakage of the superficial femoral artery after injection of contrast medium under fluoroscopic control. A false aneurysm $1 \times 2 \mathrm{~cm}$ in size was detected on the common femoral vein. This was assumed to be responsible for the symptoms. It was fixed by a figure of 8 Prolene suture.

After the operation, the patient was instructed to rest, and preventive antithrombotic drugs were prescribed. After two weeks of bed rest combined with isometric exercises, he was allowed partial weight bearing with crutches. He started football specific exercise at six weeks. At his follow up at six months, he was back to his position as a first string player.

\section{DISCUSSION}

Venous aneurysms may be either congenital or acquired. Most acquired venous aneurysms develop secondary to a traumatic vascular injury..$^{2-4}$ Traumatic injury to a particular venous segment may cause disruption and weakness in the vessel wall and gradually result in dilatation and aneurysm formation, either true or false. Peripheral venous aneurysms may be seen in both lower and upper extremities and most have little clinical significance. ${ }^{7}$ However, they may present with severe clinical pictures such as deep venous thrombosis and pulmonary embolism. ${ }^{8}$

DSA produced a false positive result in this case. The wrong preoperative diagnosis in this case may be a result of problems attributable to angiography. Like almost every other diagnostic modality, it has user and technique dependent aspects to it. An error in the chain of consecutive steps may have resulted in the misdiagnosis. The premature diagnosis based on colour Doppler ultrasound and early DSA images may have been altered by DSA examination at a later stage. Simultaneous traumatic injury to the superficial femoral artery and the common femoral vein may be another explanation. The arterial pseudoaneurysm may have collapsed by the time of the operation and left behind the false venous aneurysm as the sole pathology.

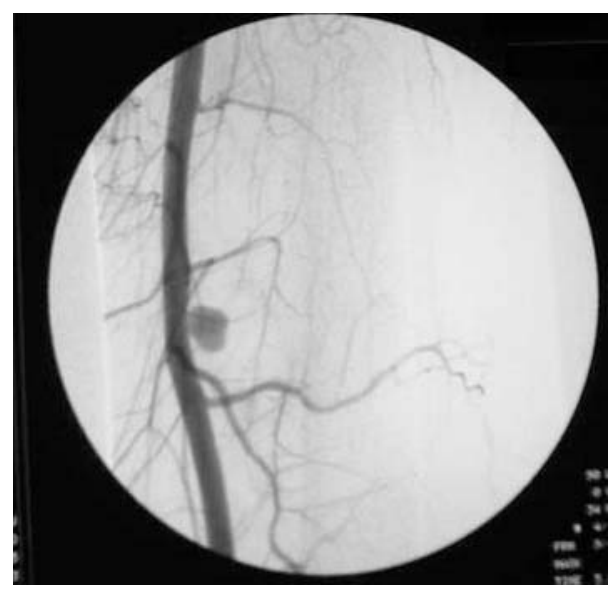

Figure 1 Left superficial femoral artery digital subtraction angiography examination, right oblique view. An anterolaterally oriented pseudoaneurysm can be seen. 
The patient's symptoms suggested a venous injury. Massive swelling with exercise and excruciating pain that resolves with rest are symptoms that should have persuaded us to include false venous aneurysm in our differential diagnosis. Unfortunately, venous aneurysms may be missed in both clinical and radiological evaluations of trauma patients under the current restricted literature back up. We believe that failure to precisely diagnose this case before surgery was due to its rare occurrence.

The symptoms of the patient were due to leakage of blood from the false femoral venous aneurysm during heavy exercise. He had no symptoms of leakage during rest, which means that the vessel wall was able to withstand venous pressure during rest. Therefore we may have prescribed rest for this patient if we had known that it was a venous injury.

In conclusion, this is the first reported case of a posttraumatic false aneurysm of the femoral vein. It may appear to be an arterial aneurysm and lead to a surgical procedure. Late phase images of DSA should always be obtained as conclusive evidence in diagnosing arterial pathology. Although this is an extremely uncommon occurrence, it is an important diagnostic consideration when examining a patient with a post-traumatic swelling.

\section{Authors' affiliations}

M Karahan, Department of Orthopedic and Traumatology, Marmara University School of Medicine, Istanbul, Turkey
S Isbir, Department of Cardiovascular Surgery, Marmara University School of Medicine

F Baltacýoglu, Department of Radiology, Marmara University School of Medicine

M M Caglar, Sportomed, Physical Therapy Institute, Istanbul

Competing interests: none declared

Correspondence to: Associate Professor Karahan, Department of Orthopedics, Mamara University School of Medicine, Kuzu Sok 3/2 Fatma Karaca Apt, Saskinbakkal, Kadikoy, Istanbul 81070, Turkey; drmustafakarahan@yahoo.com

Accepted 22 August 2004

\section{REFERENCES}

1 Legnani G, Metini L, Bonfioli C, et al. Venous aneurysm of extremities: a case report and literature review. Cardiovasc Surg 1995;3:445-6.

2 Risse M, Reuhl J, Ogbuihi S, et al. Traumatic venous aneurysm of the popliteal vein with outcome: a case report and review of the literature. J Forensic Sci 2001;46:1492-7.

3 Paes T, Andrews S, Wyatt A. Acquired venous aneurysms. Br J Sports Med 1991;25:149-50.

4 Aprin H, Schwartz GB, Valderamma E. Traumatic venous aneurysm. Case report. Clin Orthop 1987; 127:243-6.

5 Weinstein RN. False aneurysm presenting as delayed posterior interosseus nerve palsy. J Orthop Trauma 1996;10:583-5.

6 Menon KR, Schilders E, O'Connor $\mathrm{P}$, et al. Traumatic false aneurysm of a saphenous vein tributary in a cricketer. Am J Sports Med 2003;31:1017-18.

7 Browse NL, Burnand KG, Thomas ML. Diseases of the veins: pathology, diagnosis, and treatment. In: Browse NL, Burnand KG, Thomas ML, eds. Venous injury. London: Edward Arnold, 1988:645-8.

8 Sigg P, Koella Ch, Stobe Ch, et al. Popliteal venous aneurysm, a cause of pulmonary embolism. Vasa 2003;32:221-4. 